\section{Ear disorders in children}

\author{
MARGARET REICH \\ Senior Staff Editor
}

Like sticky fingers, runny noses, and scraped knees, ear infections are indigenous to childhood. In fact, only the common cold and sore throats pose more prevalant pediatric problems.

Children are particularly susceptible to outer and middle ear infections (otitis externa and otitis media) in part because they tend to poke and prod their ears. This invites bacteria to set up house in the broken skin lining the ear canal. Foreign objects such as fingers or pencils poked into the ear can further upset normal anatomical functions, causing inflammation or fluid retention.

\section{Anatomy}

\section{Outer ear}

The ear is involved with both hearing and balance. As the figure on page 285 illustrates, it is divided into three sections - outer, middle, and inner. Shaped like a funnel, the outer ear consists of visible skin folds and cartilage (pinna). This cartilage is covered with skin that produces glands that secrete wax (cerumen). The outer canal also is lined with tiny hair follicles. Both the wax and the hair follicles protect the ear.

\section{Middle ear}

Separating the outer from the middle ear is the eardrum or tympanic membrane. Three small, connected bones-hammer, anvil, and stirrup-are located here. In addition, several openings, one of which leads to the mastoid region of the temporal bone (the curved area just behind the ear containing internal ear structures), comprise the middle ear. Chief among these is the auditory (eustachian) tube which leads to the opening at the back of the nose. Equal pressure inside and outside the eardrum is maintained because of the auditory tube.

\section{Inner ear}

The snail-like cochlea, lined with hairs and filled with fluid, receives magnified sound vibrations from the eardrum that stimulate the hair follicles. These, in turn, signal the brain by way of auditory nerves to "hear sound."

Also in the inner ear is the labyrinth which is composed of three connected tubes bent into semicircles. It provides balance.

There are many problems that affect the ear. While middle ear infections tend to be more serious, common outer ear disorders include "swimmer's ear" and the accumulation of ear wax, both of which can affect your child's hearing and, ultimately, learning skills. Even foreign objects lodged inside the ear can pose a problem if not properly removed.

\section{Outer ear disorders}

\section{"Swimmer's ear"}

External otitis, also known as "swimmer's ear," is as much a part of the hot-weather season for children as a day spent swimming at the beach. Heat and moisture provide the prime breeding conditions for bacteria that cause this familiar disorder. Anything that retains moisture inside the ear, such as swimming caps or radio earplugs left in place for an extended period, invites bacteria to invade the outer ear skin, which, if left untreated, can cause infection.

Water is not the only culprit, however. Hair spray or dye you may use to style your child's hair for Halloween or the school play can also provoke this disorder. Before applying these products, insert lambswool balls into the ears. Unlike cotton, lambswool repels liquid.

\section{Symptoms}

Symptoms that external otitis may be present can appear as early as 6 hours after prolonged swimming, or up to 5 days after the aquatic experience. Initial symptoms are an itching and a plugged feeling usually due to swelling in the ear. Keeping the canal dry and refraining from inserting any objects or fingers inside the ear will enable the 
symptoms to subside, usually without medical attention.

If the ear canal remains moist, however, what initially began as a mild case of external otitis can escalate to a swollen canal. As this infection worsens, a blue-gray discharge can ensue, accompanied by intense pain that is aggravated by jaw movement.

\section{Treatment}

Aspirin and a warm clean cotton or heating pad will help relieve pain until you're able to take your child to the doctor. Professional attention involves examination with an otoscope at which time your physician will probably take a pus sample if present. Cleaning the canal is necessary to reduce pain and itching as well as to restore the canal lining.

Your physician can readily treat mild disorders by drying the outer ear with small pieces of cotton, while the more severe inflamed infection will require suctioning out the cheesey-like secretions.

In addition, an ear wash consisting of one part vinegar to one part water or rubbing alcohol may be applied, after which the canal should be dried thoroughly. In severe instances of external otitis, such irrigation methods can and should be repeated daily at home until the inflammation subsides.

Ear drops containing alcohol, or in cases where the eardrum is perforated, antibiotics are administered to clear up the infection. A homemixture of equal amounts of vinegar and rubbing alcohol has been proven as effective as commercial preparations in treating simple cases of "swimmer's ear." Drops should be left in at least 2-3 minutes.

If the canal is inflamed so much that drops cannot penetrate the area, the physician may insert a small piece of cotton laced with a water-based medicine. About 2-4 days later you can remove the cotton strands using tweezers. Ear drops should then be administered as described above. Remember to gently position the ear so the drops travel to the deepest area of infection.

Once treatment begins, your child should stay out of the water at least 1 week depending on the extent of pain, inflammation, and infection. However, daily baths or showers are permissible. Hair may be shampooed every other day as long as ears are thoroughly dried afterwards, followed by administration of the aforementioned drops.

\section{Preventing recurrence}

Although it's difficult to determine the exact time your external otitis prone child should be allowed in the water, try to set a time limit for such activities. For most susceptible children, no more than 1 hour at a stretch should be spent in the water.

Give ears a chance to dry out-at least 1 to 2 hours - in between swims. If your child feels water in the ear, have him or her shake it out and immediately towel dry the ear.

Finally, if these precautions do not prevent this condition from recurring, apply a few of those watervinegar or vinegar-rubbing alcohol ear drops in the morning, before a swim, and just before bed. Keep the solution in the ear about 5 minutes for each application.

\section{Ear wax accumulation}

Made of the frothy part of hardened protein and mixed with tiny hair follicles, wax serves to protect and clean the ear. Normally, it forms on the bottom of the canal where cartilage is present. The hair lining the canal then carries the old wax and debris along to the outside opening.

Sometimes, the wax ends up blocking the canal opening. When that happens, a ringing or plugged sensation in the ear and even partial hearing loss may occur.

Do not use a cotton swab to clean excess cerumen as it only serves to pack it against the eardrum, which might cause damage. Your physician can carefully clean this build-up by using drops to soften it before removal. In stubborn cases, a probe or electronic device may be necessary. Often, warm water directed at the cerumen will suffice.

Such accumulation is not limited to children. Because each person produces varying amounts of wax, anyone can suffer from build-up. Likewise, the cerumen color can range from yellow-gold to black, especially in older persons. Consistency differs from person to person, too, from soft and oily to hard, rice-like deposits. 


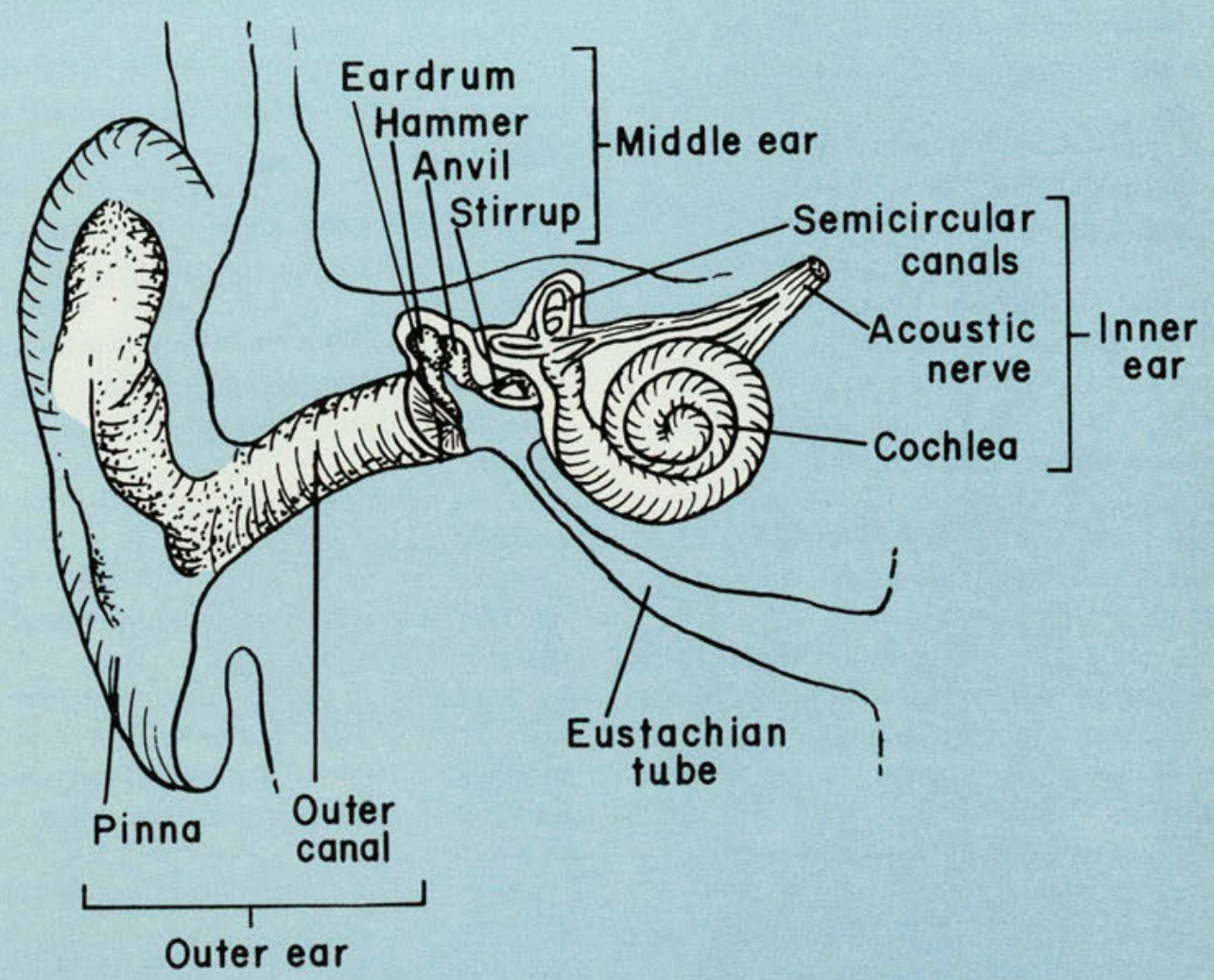

\section{Foreign objects}

Small objects (beads, earrings, popcorn kernels) lodged in the ear, are a common, if somewhat embarrassing, occurrence. Should anything out of the ordinary find its way into your ear, it's best to have a physician remove the offending agent to avoid any possible damage to the canal, eardrum, or other internal structures. If an insect buzzes into the ear, several drops of mineral oil should immobilize the little bugger long enough so that your physician can remove it.

\section{Otitis media}

Usually caused by a virus, bacteria, or a nasal allergy, inflammation and infection of the middle ear (otitis media) is especially common among preschoolers. By the time most children reach age 3, they will have had at least one such incident.

Symptoms begin much the same way as in external otitis but usually include fever accompanied by a sharp, piercing pain in the ear. Your child may appear inattentive and have difficulty hearing. He or she may tug and scratch the ear frequently, a strong indication that fluid is present in the ear.

Excessive middle ear fluid is usually the result of an abnormal fluid production or blockage of the auditory (eustachian) tube. Especially in the latter instance, inflammation will likely ensue, another hallmark of an infection.

\section{Blockage}

Auditory tube

The reason most middle ear disorders occur before the age of 4 may be partly attributed to the narrow 
opening and short length of the eustachian tube along with an underdeveloped tensor veli palatini, the muscle responsible for opening and closing the auditory tube.

This channel keeps nose and throat mucus out of the middle ear, particularly the tympanic membrane, in addition to maintaining equalized pressure. When it is not functioning properly, the chance of a middle ear infection increases due to possible bacteria present in the mucus.

In rare instances, even when the eustachian tube is fully mature, it may be blocked due to growth of adenoids. These gland-like structures, located at the back of the nose just above the tonsils, act as a protective measure against respiratory infections in children under age 5 . As the child grows, the adenoids normally shrink in size.

However, if they become enlarged, the airway to the nose and throat, as well as the eustachian tube, may be blocked. Chronic fluid in the middle ear can carry over into adulthood due to enlarged adenoids, requiring surgical removal.

Hay fever is also suspected in playing an indirect role in middle ear disorders. Allergic reactions produce histamines, chemicals that cause fluid retention and inflammation in the sinuses and nasal passage. As with enlarged adenoids, this excess fluid coupled with inflammation may block the eustachian tube, serving as a first step toward middle ear infection.

\section{Treatment}

Antibiotics, such as amoxicillin, help clear up any bacterial infection. However, they do not promote drainage and ventilation, especially important in chronic cases of otitis media, where any hearing loss can threaten your child's speech and language development.

\section{Drainage}

Antihistamines and nasal decongestants usually suffice. However, in chronic instances, drainage and ventilation are achieved by myringotomy. In this procedure, usually performed by an ear specialist (otolaryngologist), an incision is made in the eardrum. The fluid is then suctioned out of the middle ear.
If this method proves unsuccessful, tiny typanostomy tubes may be inserted in the eardrum for ventilation. Implanted on an in- or out-patient basis, these tubes may remain in place for 7 to 9 months.

Children with open typanostomy tubes should take care not to get any water in their ears. That means avoiding high diving and submerging their heads under more than 2 feet of water. Should any complications arise, such as pus draining from the ear, or if the tubes should spontaneously pop out from the eardrum, see your physician as soon as possible.

A less drastic approach for hay-fever sufferers prone to middle ear disorders may involve avoiding certain foods or areas that provoke an allergic reaction, and with it promote the possibility of eustachian tube blockage.

As a parent, it's important to realize that most ear disorders heal without medication. And while recognizing their symptoms won't solve all childhood crises, it should make those ear-related ones easier to handle.

(All material in the Patient Health Guide is reviewed by JAOA Physician referees.)

\section{References:}

Acute otitis media-Does therapy alter its course? By Moshe Frenkel. Postgraduate Medicine, vol 82, p 84, October 1987

A practical approach to chronic otitis. By Richard H. Schwartz. Patient Care, vol 20, pp 91-113, July 15, 1987.

Clinical pocket guide to ear disease. By Michael Hawke. Lea \& Febiger Philadelphia, 1987.

NASHA answers questions about otitis media and language development. National Association for Hearing and Speech Action, Rockville, Maryland, 1985.

New concepts in otitis media. By Philip Fireman. Hospital Practice, vol 22, pp 85-91, November 30, 1987.

Swimmer's ear: Timely management tips. By S. Michael Marcy. Patient Care, vol 21, pp 28-43, 82, May 30, 1987.

The American Medical Association Family Medical Guide. Edited by leffrey RM Kunz, pp 328, 330-32, 667, Random House, New York, 1982.

The Columbia University College of Physicians and Surgeons Complete Home Medical Guide. Edited by David F. Tapley, et al, pp 223, 674-78 Crown Publishers, Inc, New York, 1985. 


\section{There's more to growth than just getting bigger}

Growth and development in infants is measured in ways besides length and weight.

More sensitive criteria are behavioral development and metabolic measures such as fat absorption, bone mineralization, plasma trace minerals and plasma amino acids.

With Similac ${ }^{\circledR}$ Infant Formulas you can be confident of research documenting closeness to breast milk in many measures of growth.

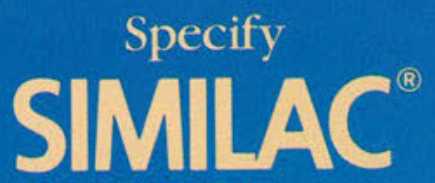

Infant Formulas 


\section{ANNOUNCING}
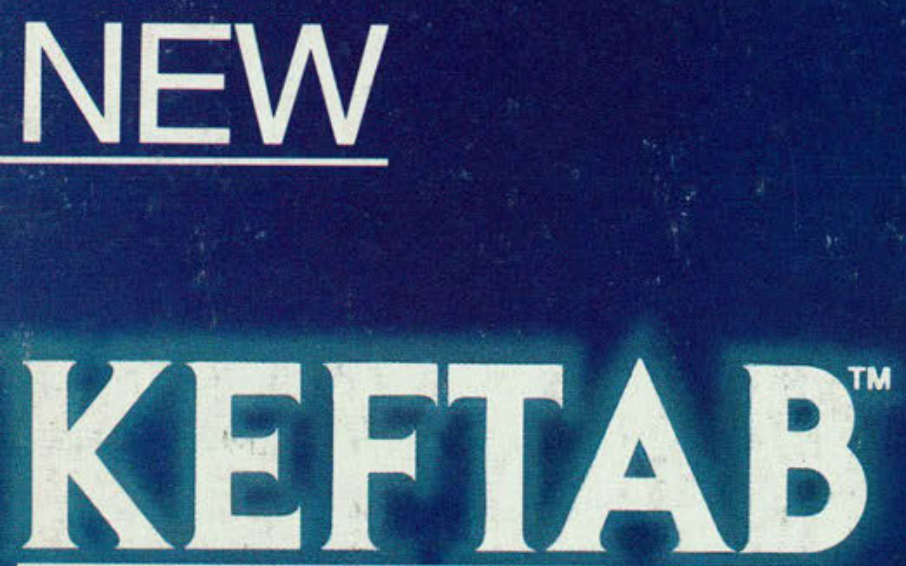

cephalexin hydrochloride monohydrate

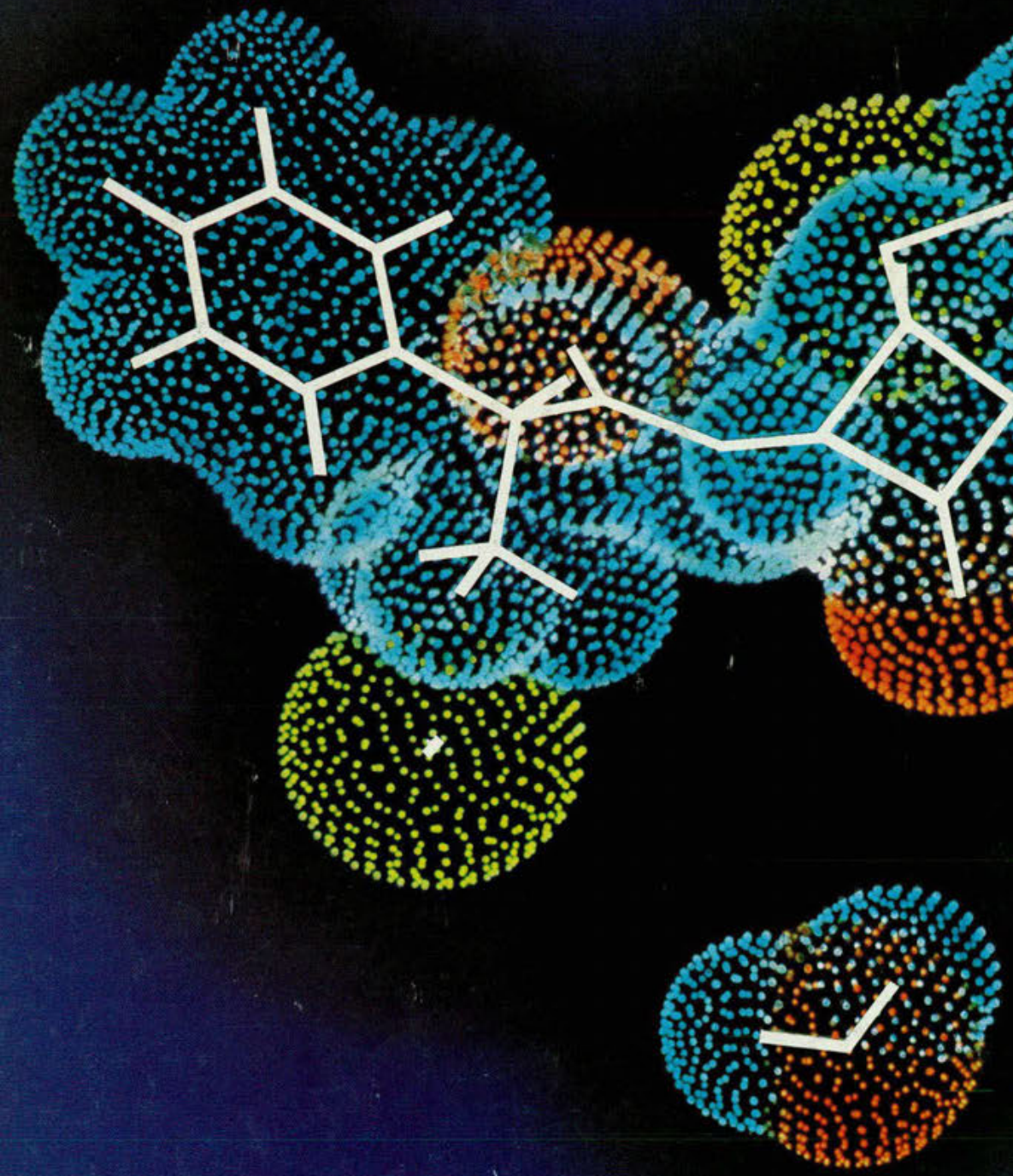

